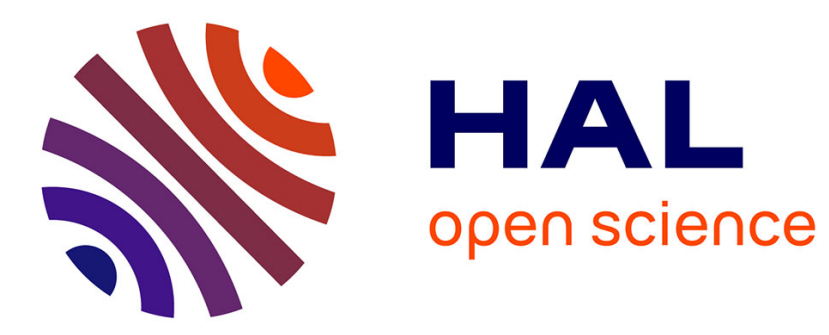

\title{
Démographie et fluctuations économiques
}

Hippolyte d'Albis, Emmanuelle Augeraud-Véron, Marie Bessec

\section{To cite this version:}

Hippolyte d'Albis, Emmanuelle Augeraud-Véron, Marie Bessec. Démographie et fluctuations économiques. Revue Economique, 2004, 55 (3), pp.429-437. hal-00630246

\section{HAL Id: hal-00630246 https://hal.science/hal-00630246}

Submitted on 7 Oct 2011

HAL is a multi-disciplinary open access archive for the deposit and dissemination of scientific research documents, whether they are published or not. The documents may come from teaching and research institutions in France or abroad, or from public or private research centers.
L'archive ouverte pluridisciplinaire HAL, est destinée au dépôt et à la diffusion de documents scientifiques de niveau recherche, publiés ou non, émanant des établissements d'enseignement et de recherche français ou étrangers, des laboratoires publics ou privés. 


\author{
Hippolyte d'Albis ${ }^{1}$ \\ Emmanuelle Augeraud-Véron ${ }^{2}$ \\ Marie Bessec ${ }^{3}$
}

\begin{abstract}
Cet article montre qu'un modèle néo-classique, aux hypothèses habituelles mais pourvu d'une structure par âge de la population explicite, converge vers son sentier de croissance équilibré avec des oscillations amorties. Il reproduit ainsi un processus de retour à la moyenne avec fluctuations de court terme que l'on observe dans les séries de produit intérieur brut de la plupart des pays de l'OCDE.
\end{abstract}

\title{
DEMOGRAPHY AND ECONOMIC FLUCTUATIONS
}

This paper shows that a neoclassical model with standard assumptions but endowed with an explicit age structure of the population, converges to its balanced growth path with damped oscillations. It therefore reproduces a trend-reverting process with short run fluctuations that is displayed by the gross domestic product time series of most OECD countries.

Classification JEL : D50, D90.

\section{INTRODUCTION}

Cet article analyse l'impact de la structure par âge de la population sur la dynamique de l'économie. L'idée de ce travail provient d'un article récent de Azariadis, Bullard et Ohanian [2004]. Ils conjecturent qu'un modèle doté d'un grand nombre de générations imbriquées devrait pouvoir reproduire les fluctuations de court terme observées dans les séries de PIB des Etats-Unis. Ils développent leur argument théorique à partir de Kehoe, Levine, Mas-Colell et Woodford [1991] qui considèrent une économie d'échange de type Gale [1973] et supposent la substituabilité brute des fonctions de demande. Dans un tel cadre, Azariadis et al. prouvent que la convergence des prix vers l'équilibre stationnaire n'est pas monotone. Ils étudient ensuite une économie avec production et montrent, à l'aide d'une simulation numérique, que la convergence se fait par oscillations amorties. Aussi, un modèle néoclassique déterministe à un seul secteur et avec une offre de travail inélastique semble reproduire les fluctuations du PIB s'il est doté d'une structure démographique réaliste. Il n'est

\footnotetext{
${ }^{1}$ EUREQua, Université Paris-I. 106-112, boulevard de l'Hôpital, 75647 Paris cedex 16. dalbis@univ-paris1.fr

${ }^{2}$ LMA, Université de la Rochelle et EUREQua. Avenue Michel Crépeau, 17042 La Rochelle. eaugerau@univlr.fr

${ }^{3}$ EURISCO, Université Paris Dauphine. Place du Maréchal de Lattre de Tassigny, 75775 Paris cedex 16. marie.bessec@dauphine.fr
} 
bien entendu pas question de considérer la démographie comme l'unique source des fluctuations économiques mais il est intéressant de comprendre l'argument logique qui les lie.

L'objectif de notre article est d'analyser l'impact de la prise en compte de l'hétérogénéité d'âge des individus sur la dynamique des variables économiques agrégées. Nous fondons notre argumentation sur le travail de d'Albis et Augeraud-Véron [2003] qui prouvent la conjecture de Azariadis et al. lorsque la technologie agrégée est à rendement du capital constant. Nous présentons ici le mécanisme économique sans développer la démonstration mathématique.

L'objet de notre travail est également méthodologique. On observe en effet depuis Auerbach et Kotlikoff [1987], que l'utilisation de modèles avec un grand nombre de générations imbriquées est de plus en plus fréquente en macro-économie appliquée. Ces modèles ont non seulement l'avantage de faire reposer les comportements individuels sur la théorie du cycle de vie mais sont également faciles à confronter aux données statistiques et à utiliser pour l'analyse de courte période. Aussi, il est important de remarquer que les propriétés dynamiques de ces modèles sont différentes de celles des modèles à générations de type Diamond [1965] où les agents ne vivent que deux périodes. Notamment, d'Albis et Augeraud-Véron [2003] montrent que l'existence d'un équilibre dynamique n'est pas garantie mais dépend de façon cruciale de la distribution initiale de la richesse entre les générations.

Contrairement à Azariadis et al. et à une grande partie des auteurs de cette littérature, nous utilisons un modèle en temps continu proche de celui développé initialement par Cass et Yaari [1967]. Le temps continu permet, en effet, de simplifier la résolution mathématique du modèle sans en altérer les propriétés qualitatives. Demichelis et Polemarchakis [2002] ont montré que les variables endogènes d'un modèle en temps discret convergeaient de manière uniforme vers celles en temps continu lorsque la fréquence de l'échange au cours de la vie d'un individu était portée à l'infini.

Notre article est organisé de la façon suivante. Dans une première section, nous caractérisons le mouvement de retour à la moyenne du produit intérieur brut de 14 pays de l'OCDE en calculant les valeurs propres de processus auto-régressifs estimés sur les séries trimestrielles. Dans une deuxième section, nous présentons la dynamique du capital d'un modèle avec un continuum de générations imbriquées et montrons qu'elle est affectée par des délais et des avances discrets. Dans une dernière section, nous étudions cette dynamique dans le cas où les rendements du capital sont constants et expliquons pourquoi elle converge par oscillations amorties.

\section{LES OSCILLATIONS LORS DU RETOUR A LA MOYENNE}

Dans cette section, nous calculons les valeurs propres des processus auto-régressifs estimés sur les séries trimestrielles de 14 pays de l'OCDE ${ }^{4}$. Nous déterminons dans quels cas la composante cyclique est estimée par des valeurs propres complexes ou négatives.

La procédure est la suivante. On estime un processus auto-régressif univarié sur la composante cyclique du PIB réel. Cette dernière est obtenue en retranchant la tendance calculée l'aide d'un filtre Hodrick-Prescott ${ }^{5}$. On régresse alors la variable obtenue sur ses

\footnotetext{
${ }^{4}$ Nous avons utilisé des séries trimestrielles corrigées des variations saisonnières issues de la base de données en ligne de l'OCDE. Ces séries de PIB en volume (base 100 en 1995) vont du premier trimestre de 1981 au premier trimestre de l'année 2003 pour tous les pays sauf l'Allemagne pour laquelle les données commencent au premier trimestre de 1991.

${ }^{5}$ Nous avons également estimé nos modèles sur des composantes cycliques obtenues par filtrage sur une tendance linéaire et sur une tendance quadratique. Nous ne les évoquons pas ici car les résultats finaux sont moins robustes.
} 
retards et on garde le processus correspondant au dernier retard significatif à $10 \%$. On a reporté dans la seconde colonne du tableau ci-dessous ces résultats. La significativité des retards diffère grandement entre les pays. Ainsi, on utilisera des processus auto-régressifs d'ordre un pour l'Allemagne, l'Autriche et l'Italie, d'ordre deux pour la Norvège, d'ordre trois pour les Etats-Unis et d'ordre quatre pour tous les autres. On cherche alors les valeurs propres associées au processus auto-régressif retenu. On a reporté dans la troisième colonne du tableau le type de racines obtenu. On observe que tous les pays sauf l'Allemagne, l'Autriche et l'Italie ont des valeurs propres complexes ou négatives.

La robustesse des résultats a été évaluée à l'aide d'une procédure de "bootstrap ». Avec les coefficients estimés, les résidus et les valeurs initiales, on a simulé 1000 nouvelles séries par tirage avec remise dans la série de résidus. Sur chaque nouvelle série constituée, nous avons ré-estimé un processus auto-régressif et déterminé ses valeurs propres ${ }^{6}$. La quatrième colonne du tableau indique la part des cas pour laquelle la configuration des racines est la même que celle estimée sur les données initiales. On conclut à la lecture du tableau que nos résultats sont très robustes.

\begin{tabular}{|llll|}
\hline Pays & Processus & Racines & Bootstrap \\
\hline Allemagne & $\mathrm{AR}(1)$ & $1 \mathrm{R} \in(0,1)$ & 1 \\
\hline Autriche & $\mathrm{AR}(1)$ & $1 \mathrm{R} \in(0,1)$ & 1 \\
\hline Australie & $\mathrm{AR}(4)$ & 2 C p.r. $\in(0,1), 2 \mathrm{C}$ p.r. $\in(-1,0)$ & 0,943 \\
\hline Belgique & $\mathrm{AR}(4)$ & 2 C p.r. $\in(0,1), 2 \mathrm{C}$ p.r. $\in(-1,0)$ & 0,897 \\
\hline Canada & $\mathrm{AR}(4)$ & 2 C p.r. $\in(0,1), 2 \mathrm{C}$ p.r. $\in(-1,0)$ & 0,985 \\
\hline Etats-Unis & $\mathrm{AR}(3)$ & 2 C p.r. $\in(0,1), 1 \mathrm{R} \in(-1,0)$ & 0,99 \\
\hline Finlande & $\mathrm{AR}(4)$ & 2 C p.r. $\in(0,1), 2 \mathrm{C}$ p.r. $\in(-1,0)$ & 0,741 \\
\hline France & $\mathrm{AR}(4)$ & 2 C p.r. $\in(0,1), 2 \mathrm{C}$ p.r. $\in(-1,0)$ & 0,912 \\
\hline Italie & $\mathrm{AR}(1)$ & 1 R $\in(0,1)$ & 1 \\
\hline Japon & $\mathrm{AR}(4)$ & 2 C p.r. $\in(0,1), 2 \mathrm{C}$ p.r. $\in(-1,0)$ & 0,843 \\
\hline Norvège & $\mathrm{AR}(2)$ & 1 R $\in(0,1), 1 \mathrm{R} \in(-1,0)$ & 0,998 \\
\hline Pays-Bas & $\mathrm{AR}(4)$ & 2 C p.r. $\in(0,1), 2$ C p.r. $\in(-1,0)$ & 0,894 \\
\hline Royaume-Uni & $\mathrm{AR}(4)$ & 2 C p.r. $\in(0,1), 2$ C p.r. $\in(-1,0)$ & 0,823 \\
\hline Suède & $\mathrm{AR}(4)$ & 2 C p.r. $\in(0,1), 2$ C p.r. $\in(-1,0)$ & 0,723 \\
\hline
\end{tabular}

A titre d'exemple, les graphiques ci-dessous représentent les solutions pour la France et les Etats-Unis.

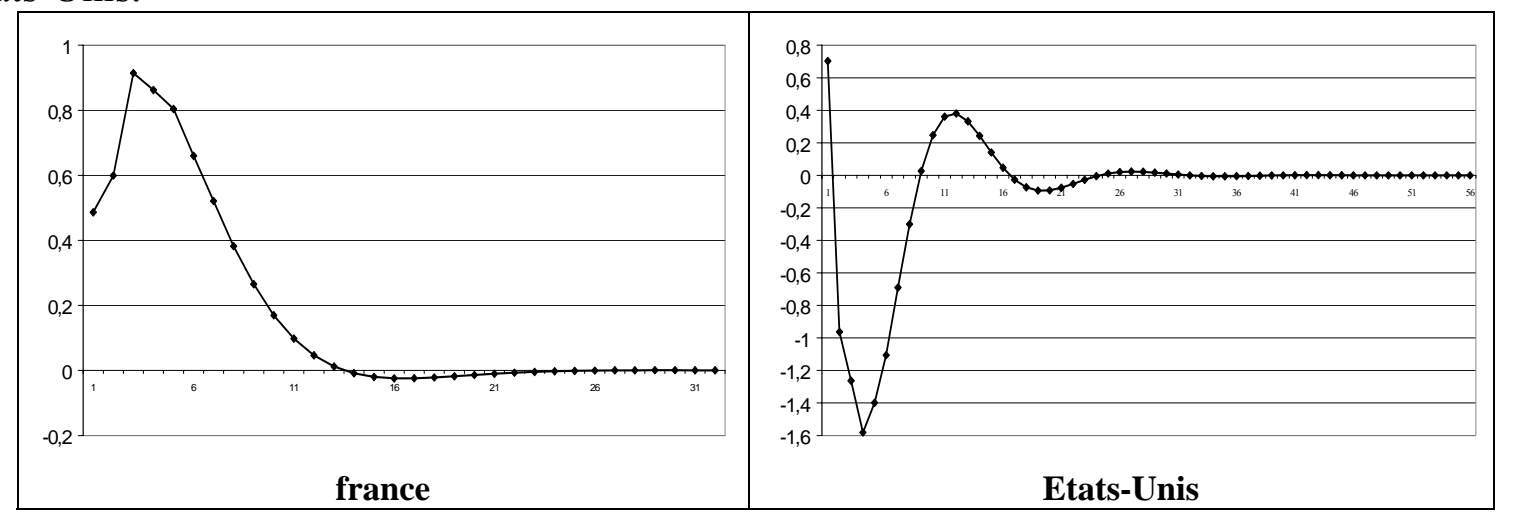

\section{UN MODELE AVEC UN CONTINUUM DE GENERATIONS IMBRIQUEES}

Dans cette section, nous déterminons la dynamique du capital issue d'un modèle à générations imbriquées en temps continu. Nous montrons que cette dynamique s'écrit sous la

\footnotetext{
${ }^{6}$ Les processus auto-regressifs sont estimés avec un nombre de retards suffisamment importants pour blanchir les résidus. Il n'est donc pas nécessaire de recourir à des procédures de bootstrap par bloc.
} 
forme d'une équation différentielle avec délais et avances et discutons les hypothèses qui les génèrent.

Le modèle se construit en deux temps. Premièrement, la trajectoire de consommation individuelle est établie à partir d'un comportement inter-temporel de cycle de vie. Ensuite, les décisions individuelles sont agrégées dans une structure par âge de la population et la dynamique de la consommation globale est déterminée.

Chaque individu a une durée de vie certaine et finie égale à $\omega$. Son programme est de déterminer la trajectoire de consommations positives qui maximise son utilité inter-temporelle sous une contrainte de budget. On suppose que la fonction objectif, notée V(s), d'un individu né à la date $\mathrm{s}$ après la date initiale de l'économie, est une somme escomptée de fonctions d'utilité instantanée de type CRRA telle que :

$$
\mathrm{V}(\mathrm{s})=\int_{\mathrm{s}}^{\mathrm{s}+\omega} \exp (-\rho(\mathrm{z}-\mathrm{s})) \frac{\mathrm{c}(\mathrm{s}, \mathrm{z})^{1-1 / \sigma_{-1}}}{1-1 / \sigma} \mathrm{dz}
$$

où $\mathrm{c}(\mathrm{s}, \mathrm{z})$ est la consommation à l'âge $(\mathrm{z}-\mathrm{s}), \sigma>0$ est l'élasticité de substitution intertemporelle et $\rho$ est le taux de préférence pour le présent. Au cours de sa vie, l'individu offre de manière inélastique une unité de travail et bénéficie d'un salaire qui peut dépendre de son âge et qui est noté $\mathrm{w}(\mathrm{s}, \mathrm{z})$. De plus, il a accès à des marchés financiers compétitifs qui rémunèrent son épargne au taux d'intérêt $\mathrm{r}(\mathrm{z})$. La contrainte budgétaire inter-temporelle s'écrit alors :

$$
\int_{\mathrm{s}}^{\mathrm{s}+\omega} \exp \left(-\int_{\mathrm{s}}^{\mathrm{z}} \mathrm{r}(\mathrm{u}) \mathrm{du}\right) \mathrm{c}(\mathrm{s}, \mathrm{z}) \mathrm{dz} \leq \int_{\mathrm{s}}^{\mathrm{s}+\omega} \exp \left(-\int_{\mathrm{s}}^{\mathrm{z}} \mathrm{r}(\mathrm{u}) \mathrm{du}\right) \mathrm{w}(\mathrm{s}, \mathrm{z}) \mathrm{dz}
$$

La maximisation de (1) sous la contrainte (2) détermine la consommation optimale de l'individu. Sa dynamique est donnée par la condition d'Euler suivante :

$$
\frac{\partial \mathrm{c}(\mathrm{s}, \mathrm{t})}{\partial \mathrm{t}}=\sigma[\mathrm{r}(\mathrm{t})-\rho] \mathrm{c}(\mathrm{s}, \mathrm{t})
$$

tandis que le niveau initial de sa consommation est obtenu en remplaçant (3) dans (2). Après quelques manipulations, on obtient l'expression suivante de la consommation initiale :

$$
\mathrm{c}(\mathrm{s}, \mathrm{s})=\int_{\mathrm{s}}^{\mathrm{s}+\omega} \exp \left(-\int_{\mathrm{s}}^{\mathrm{z}} \mathrm{r}(\mathrm{u}) \mathrm{du}\right) \mathrm{w}(\mathrm{s}, \mathrm{z}) \mathrm{dz} / \int_{\mathrm{s}}^{\mathrm{s}+\omega} \exp \left(-\int_{\mathrm{s}}^{\mathrm{z}}[(1-\sigma) \mathrm{r}(\mathrm{u})+\rho] \mathrm{du}\right) \mathrm{dz}
$$

On suppose que chaque individu né à la date s appartient à une cohorte d'individus identiques de taille $b(s)$. A chaque instant, une nouvelle cohorte apparait tandis que celle ayant atteint l'âge $\omega$ disparaît. La consommation agrégée à la date $t$ est donc déterminée en intégrant les consommations individuelles sur les dates de naissance :

$$
C(t)=\int_{t-\omega}^{t} b(s) c(s, t) d s
$$

On remarque avec l'équation (4) que l'ensemble des valeurs du taux d'intérêt et du salaire sur l'intervalle $[\mathrm{t}-\omega, \mathrm{t}+\omega]$ influence le niveau de la consommation agrégée à la date $\mathrm{t}$. On remarque que si l'économie commence à une date finie, les consommations individuelles des individus nés avant cette date dépendent d'une dotation initiale exogène. La consommation agrégée dépend alors des dotations initiales tant que ces individus sont en vie.

En différentiant par rapport au temps l'équation (5), et en utilisant l'équation (3), on obtient la dynamique de la consommation agrégée suivante :

$$
\frac{d C(t)}{d t}=b(t) c(t, t)-b(t-\omega) c(t-\omega, t)+\sigma[r(t)-\rho] C(t)
$$

L'équation (6) diffère de celle du modèle canonique de type Ramsey par la présence des deux premiers termes de son membre de droite. Le troisième terme pris isolément reflète en effet le taux de croissance de la consommation d'une économie peuplée d'une unique dynastie à durée de vie infinie. Le terme $b(t) c(t, t)$ marque l'influence de l'entrée d'une nouvelle cohorte 
sur la dynamique de la consommation agrégée. Avec Weil [1989], on interprète ce terme comme la mesure de l'absence d'altruisme entre les générations. On remarque avec (4) que ce terme dépend de l'ensemble des valeurs du taux d'intérêt et du salaire sur l'intervalle $[\mathrm{t}, \mathrm{t}+\omega]$. Symétriquement, le terme $b(t-\omega) c(t-\omega, t)$ marque l'influence, sur la dynamique de la consommation agrégée, du «départ» de la génération la plus âgée. Cette fois, c'est l'ensemble des valeurs du taux d'intérêt et du salaire sur l'intervalle $[\mathrm{t}-\omega, \mathrm{t}]$ qui compte.

On suppose que la production est décrite par le comportement d'une firme produisant un bien unique en utilisant du capital et du travail. Elle détermine ses demandes de facteurs en situation de concurrence. L'optimum du producteur est atteint lorsque le prix de chaque facteur est égal à sa productivité marginale. On en déduit, pour chaque date $t$, la relation entre les prix des facteurs et le stock de capital à la date $t$, noté $K(t)$. L'hypothèse d'anticipations rationnelles de la part des individus permet dès lors de réécrire l'équation (5) sous la forme suivante :

$$
\mathrm{C}(\mathrm{t})=\mathrm{F}(\mathrm{K}(\mathrm{z}), \mathrm{z} \in[\mathrm{t}-\omega, \mathrm{t}+\omega])
$$

A l'équilibre du marché des biens, tel que la production est égale à la somme de la consommation agrégée et de l'investissement, on a donc une équation différentielle en $\mathrm{K}(\mathrm{t})$ qui satisfait la forme suivante :

$$
\frac{\mathrm{dK}(\mathrm{t})}{\mathrm{dt}}=\mathrm{G}(\mathrm{K}(\mathrm{z}), \mathrm{z} \in[\mathrm{t}-\omega, \mathrm{t}+\omega])
$$

La dynamique du stock de capital à la date $\mathrm{t}$ dépend donc de l'ensemble de ses valeurs sur l'intervalle $[t-\omega, t+\omega]$. Les termes prenant valeur sur $[t-\omega, t)$ sont appelés les délais tandis que les termes sur $(t, t+\omega]$ sont les avances. Les délais sont dus à l'hypothèse de durée de vie finie et disparaissent dans les modèles à générations imbriquées de type Blanchard [1985] et Weil [1989]. Les avances sont dues aux hypothèses jointes d'absence d'altruisme intergénérationnel et d'anticipations parfaites.

Les équations différentielles avec délais ${ }^{7}$ ont été utilisées pour décrire des phénomènes économiques dès Frisch [1933] et Kalecki [1935]. Elles ont connu un regain d'intérêt avec Benhabib et Rustichini [1991] et les modèles à générations de capital. Dans ce dernier cas, comme le relèvent Boucekkine, de la Croix et Licandro [2002], c'est également l'hypothèse de durée de vie finie, même s'il s'agit de celle du capital, qui est à l'origine des délais dans la dynamique. En revanche, la dynamique globale d'une équation différentielle contenant à la fois des délais et des avances n'avait pas été étudiée dans la littérature économique. Dans la section suivante, nous présentons la solution dans le cas d'une technologie à rendements constants du capital.

\section{LE CAS DES RENDEMENTS CONSTANTS DU CAPITAL}

Dans cette section, nous proposons une explication théorique du phénomène de retour à la moyenne observé dans la première section. Pour cela nous reprenons l'argument développé par d'Albis et Augeraud-Véron [2003] sans détailler les calculs du modèle. Nous présentons d'abord la démarche de résolution puis insistons sur l'intuition économique.

L'équation dynamique du capital donnée en (8) est généralement non linéaire. Cependant, si on utilise une fonction de production avec externalité de capital de type « apprentissage par la pratique » telle que :

$$
\mathrm{Y}(\mathrm{t})=\mathrm{A}(\mathrm{K}(\mathrm{t}))^{\alpha}(\mathrm{L}(\mathrm{t}) \mathrm{e}(\mathrm{t}))^{1-\alpha}
$$

\footnotetext{
${ }^{7}$ Voir Bellman et Cooke [1963] pour une introduction.
} 
où $\mathrm{e}(\mathrm{t})=\mathrm{K}(\mathrm{t})$ est l'externalité, le modèle est linéaire. En outre, $\mathrm{L}(\mathrm{t})$ représente le travail et on pose $0<\alpha<1$ et $\mathrm{A}>0$. A l'équilibre, on obtient un taux d'intérêt constant et un salaire proportionnel au stock de capital. On en déduit, avec les équations (4) et (5), que la consommation agrégée est linéaire par rapport au capital et donc que l'équation différentielle régissant la dynamique du capital l'est également. Avec cette hypothèse, on obtient un modèle de croissance endogène dont on étudie l'équilibre concurrentiel dynamique. On montre que, si elle existe, la solution de l'équation différentielle s'écrit de la façon suivante :

$$
K(t)=\sum_{n} \delta_{n} \exp \left(g_{n} t\right)
$$

où les $\delta_{\mathrm{n}}$ sont des constantes et où les $\mathrm{g}_{\mathrm{n}}$ sont les solutions de l'équation caractéristique associée à l'équation différentielle.

Comme le remarque Rustichini [1989], la présence simultanée d'avances et de retards rend la question de l'existence d'une solution à l'équation différentielle non triviale car le problème de Cauchy est mal posé.

Par ailleurs, poser le problème de l'initialisation implique que l'économie débute à une date finie, $\mathrm{t}=0$. Cela revient à poser la distribution de la richesse financière entre les générations à cette date initiale. Par conséquent, chaque individu né avant la date initiale, est pourvu d'une dotation financière qui n'est pas le produit d'une accumulation optimale mais qui représente l'une des conditions initiales de l'équation différentielle. La dynamique est alors définie en distinguant l'évolution de l'économie pour $t \in[0, \omega]$ tant qu'il existe des générations nées avant la date initiale, de celle qui prévaut pour $t>\omega$; la dynamique est donc définie par morceaux. Il est alors possible d'exprimer la forme de la distribution de la richesse financière en fonction des $\delta_{\mathrm{n}}$ et des $\mathrm{g}_{\mathrm{n}}$ et de montrer qu'il existe des distributions initiales telles qu'il n'existe pas de solution à l'équation différentielle. En revanche, s'il existe une solution, elle est unique.

Le comportement dynamique du capital dépend donc des racines $\mathrm{g}_{\mathrm{n}}$ de l'équation caractéristique. On montre qu'il existe une unique racine réelle pure notée $\hat{g}$, une infinité de racines complexes à partie réelle strictement supérieure à $\hat{g}$ et une infinité de racines complexes à partie réelle strictement inférieure à $\hat{g}$.

La racine $\hat{g}$ correspond au sentier de croissance équilibré. On montre que ce taux de croissance est nécessairement inférieur au taux d'intérêt exogène, $\alpha \mathrm{A}$. Cette propriété est nécessaire pour avoir un stock de capital strictement positif. En effet, si le taux de croissance du revenu non financier est supérieur au taux de rendement de l'épargne, il est possible d'être endetté toute sa vie. On constate également qu'il n’y a pas de racines complexes à partie réelle égale à $\hat{g}$, ce qui élimine la possibilité de cycles auto-entretenus.

En fonction des racines complexes, on va alors distinguer deux types de trajectoires: soit la dynamique du capital converge vers son sentier de croissance équilibré, soit elle diverge. En effet, toute solution qui comprend au moins une racine complexe à partie réelle supérieure à $\hat{g}$ se caractérise par des oscillations d'amplitude de plus en plus grande et viole nécessairement avant une date finie la contrainte de positivité du capital. Inversement, toute solution dont les racines complexes sont à parties réelles inférieures à $\hat{g}$ converge vers le sentier de croissance équilibré. La solution est donc nécessairement caractérisée par des fluctuations exponentiellement décroissantes. Si on note les racines $g_{n}=p_{n}+i q_{n}$, la solution s'écrit donc :

$$
\mathrm{K}(\mathrm{t})=\delta_{1} \exp (\hat{\mathrm{g}} \mathrm{t})+\sum_{\mathrm{p}_{\mathrm{n}}<\hat{\mathrm{g}}} \delta_{\mathrm{n}} \exp \left(\mathrm{p}_{\mathrm{n}} \mathrm{t}\right) \cos \left(\mathrm{q}_{\mathrm{n}} \mathrm{t}\right)
$$

Enfin, le taux de croissance endogène du capital est caractérisé par une trajectoire de type point-selle. La trajectoire solution converge vers l'état stationnaire $\hat{g}$ tandis que les autres divergent. 
Il convient de noter avec Burke [1990] que l'hypothèse d'une date initiale à l'économie est cruciale. On comprend que si l'économie existe depuis un temps infini, les solutions avec oscillations exponentiellement décroissantes violent également la contrainte de positivité du capital. Dans ce cas, l'économie est directement sur son sentier de croissance équilibré. La présence de fluctuations au cours de la transition dépend donc d'une distribution non stationnaire de la richesse financière au sein de la population à une date initiale donnée.

Quel mécanisme explicatif notre modèle propose-t-il à ces fluctuations ? L'argument repose sur l'effet d'écho bien connu des modèles à générations de capital. Le mécanisme est le suivant: supposons que l'économie soit sur un sentier de croissance équilibré et que survient un accroissement non anticipé de la part du capital dans la valeur ajoutée. Chaque individu en vie au moment du choc voit donc le taux de croissance exogène de sa consommation brutalement augmenter. Il doit donc, pour réaliser son plan de consommation optimal, augmenter fortement son épargne instantanée. Cette forte augmentation de l'épargne produit une rapide augmentation du stock de capital dont le taux de croissance va surpasser son état de long terme. Les générations qui naissent peu de temps après le choc bénéficient alors de salaires et d'une richesse humaine supérieurs aux niveaux de long terme. Ils sont donc moins incités à épargner, ce qui réduit le taux de croissance du capital. Le processus se reproduit en s'atténuant jusqu'à ce que le taux de croissance ait atteint son état stationnaire. On remarque, pour finir, que les dynamiques de la consommation et de l'investissement agrégés sont similaires à celles du capital.

\section{CONCLUSION}

Notre modèle montre que l'introduction d'une structure démographique dans un modèle uni-sectoriel avec des rendements du capital constants est suffisante pour générer une transition vers le sentier de croissance équilibré et donc pour éliminer une caractéristique peu satisfaisante du modèle de base avec un agent à durée de vie infinie de type Rebelo [1991]. De plus, au cours de la transition, le capital connaît des fluctuations d'amplitude décroissante similaires à celles observées à partir des données empiriques. Nos résultats dans le cadre de la croissance d'équilibre sont complémentaires de ceux que Boucekkine, Licandro, Puch et del Rio [2002] établissent dans un cadre de croissance optimale. Ils introduisent dans le modèle AK une structure par âge du capital et montrent que le taux de croissance optimal converge vers son état stationnaire avec des oscillations exponentiellement décroissantes.

Enfin, notre modèle théorique suggère que les variables anticipées influencent la dynamique du produit. Il serait intéressant de poursuivre le travail présenté dans la première section en en vue de tester empiriquement cette relation.

\section{Références Bibliographiques}

d'Albis H. et Augeraud-VÉron E. [2003], «Competitive growth in a life-cycle model: Existence and Dynamics » Cahier de la MSE $n^{\circ}$ 2004.31, Université Paris-I.

Auerbach A. et Kotlikoff L. [1987], Dynamic Fiscal Policy, Cambridge University Press, Cambridge.

AZARIADis C., Bullard J. et OHANiAn L. [2004], "Trend-reverting fluctuations in the life-cycle Model », à paraître dans le Journal of Economic Theory.

Bellman R. et CoOKE K. [1963], Differential Difference Equations, Academic Press.

BENHABIB J. et RUSTICHINI [1991], «Vintage capital, investment, and growth », Journal of Economic Theory, 55 , p. 323-339.

BouceKkine R., de la CROIX D., et LiCANDRo O. [2002], « Vintage human capital, demographic trends and endogenous growth », Journal of Economic Theory, 104, p. 340-375. 
BouceKkine R., LicAndro O., PuCh L. A. et DEL Rìo F. [2002], « Vintage capital, and the dynamics of the AK model », EUI working paper.

BLANCHARD O. [1985], « Debt, deficits and finite horizons », Journal of Political Economy, 93, p. 223-247.

BURKE J. L. [1990], «A benchmark for comparative dynamics and determinacy in overlapping-generation economies », Journal of Economic Theory, 52, p. 268-303.

CASS D., YAARI M. E. [1967], « Individual saving, aggregate capital accumulation, and efficient growth » dans Essays on the Theory of Optimal Economic Growth (K. Shell, Eds.), p. 233-268, MIT Press, Cambridge, MA.

Demichelis S., Polemarchakis H. [2002], « Frequency of trade and the determinacy of equilibrium paths: logarithmic economies of overlapping generations under certainty », Brown University Working Paper No 02-19.

DiAmond P. A. [1965], « National debt in a neoclassical growth model », American Economic Review, 55, p. 1126-1150.

FRISCH [1933], « Propagation problems and impulse problems in dynamics » dans Economic Essays in Honour of Gustav Cassel, Londres :George Allen \& Unwin, p. 171-205.

GALE D. [1973], « Pure exchange equilibria of dynamic economic models », Journal of Economic Theory, 6, p. 12-36.

KALECKI [1935], « A macrodynamic theory of business cycles », Econometrica 3, p. 327-344.

Kehoe T., Levine D., MAS-COLELl A. et WoOdFord M. [1991], « Gross substitutability in large-square economies, Journal of Economic Theory, 54, p. 1-25.

REBELo S. [1991], « Long-run policy analysis and long-run growth », Journal of Political Economy, 99, p. 500521.

RUSTICHINI [1989], «Functional differential equations of mixed type: the linear autonomous case » Journal of Dynamics and Differential Equations, 1, p. 121-143.

WeIL Ph. [1989], « Overlapping families of infinitely-lived agents », Journal of Public Economics, 38, p. 183198 . 